\title{
Basic Entropies for Positive-Definite Matrices
}

\section{Jun Ichi Fujii}

Department of Arts and Sciences (Information Science), Osaka Kyoiku University, 4-698-1 Asahigaoka, Kashiwara, Osaka 582-8582, Japan.

Received: January 21, 2015 / Accepted: February 25, 2015 / Published: April 25, 2015.

Abstract: We extend basic entropies in the classical information theory to matrix ones in the quantum information theory. Then we show that relations between matrix entropies similar to the classical ones hold.

Keywords: Matrix entropy, Information theory, Quantum entropy, Relative operator entropy

\section{Introduction}

Let $a_{j}$ and $b_{i}$ be elementary stochastic events for probability vectors $X=\left(p\left(a_{j}\right)\right)$ and $Y=\left(p\left(b_{i}\right)\right)$ for $j=1, \ldots, n$ and $i=1, \ldots, m$. Then a (classical) channel is a probability matrix $P_{c}=\left(p_{i j}\right)$ which transforms $X$ into $Y$ and the entropies in the classical information theory are defined by:

compound entropy

$$
H(X, Y)=-\sum_{i, j} p\left(a_{j}, b_{i}\right) \log p\left(a_{j}, b_{i}\right),
$$

conditional entropies

$$
\begin{aligned}
& H(X \mid Y)=-\sum_{i, j} p\left(a_{j}, b_{i}\right) \log p\left(a_{j} \mid b_{i}\right), \\
& H(Y \mid X)=-\sum_{i, j} p\left(a_{j}, b_{i}\right) \log p\left(b_{i} \mid a_{j}\right),
\end{aligned}
$$

mutual entropy

$$
I(X ; Y)=\sum_{i, j} p\left(a_{j}, b_{i}\right) \log \frac{p\left(a_{j}, b_{i}\right)}{p\left(a_{j}\right) p\left(b_{i}\right)} .
$$

The relations between them are:

$$
\begin{gathered}
H(X, Y)=H(X)+H(Y \mid X) \\
=H(Y)+H(X \mid Y)=I(X ; Y) \\
+H(X \mid Y)+H(Y \mid X),
\end{gathered}
$$

Corresponding author: Jun Ichi Fujii, Department of Arts and Sciences (Information Science), Osaka Kyoiku University, 4-698-1 Asahigaoka, Kashiwara, Osaka 582-8582, Japan. E-mail: fujii@cc.osaka-kyoiku.ac.jp.

$$
\begin{gathered}
H(X)=I(X ; Y)+H(X \mid Y), \\
H(Y)=I(X ; Y)+H(Y \mid X) .
\end{gathered}
$$

Though all entropies are non-negative, we note that the some individual mutual informations

$$
I\left(a_{j} ; b_{i}\right)=\log \frac{p\left(a_{j}, b_{i}\right)}{p\left(a_{j}\right) p\left(b_{i}\right)}
$$

may take negative values.

In this paper, we try to extend these classical information entropies to matrix ones. The key quantity is the relative entropy called the Kullback-Leibler divergence:

$$
s(X \mid Y)=\sum_{i j} p\left(a_{j}\right) \log \frac{p\left(a_{j}\right)}{p\left(b_{i}\right)} .
$$

The mutual entropy can be expressed by the relative entropy:

$$
\begin{aligned}
& I(X ; Y)=I\left(X \mid P_{c} X\right)= \\
& \mathbf{s}\left(\left(p\left(a_{j}, b_{i}\right)\right) \mid\left(p\left(a_{j}\right), p\left(b_{i}\right)\right)\right)
\end{aligned}
$$

In quantum information theory, $X$ is considered as the density matrix $A$ (i.e., $A \geq 0$ and $\operatorname{Tr} A=1$ ), the channel $P_{c}$ as a trace-preserving completely positive map $\Phi$ and the elementary events $\left\{b_{i}\right\}$ as a POVM (positive operator-valued measure) $F=\left\{F_{i}\right\}$ (i.e., $F_{j} \geq 0$ and $\sum_{j} F_{j}=I$ ). Note that an input event $\left\{a_{j}\right\}$ is considered as a PVM 
(projection-valued measure) $E=\left\{E_{j}\right\}$, that is, a decomposition of the identity. Suppose that $A=\sum_{n} t_{n} E_{n}$ is a spectral decomposition and $\Phi(A)=\sum_{n} t_{n} \Phi\left(E_{n}\right)$ where $\left\{\Phi\left(E_{i}\right)\right\}$ is a POVM. Then the Ohya mutual entropy $I(A ; \Phi)$ is defined by the Umegaki relative entropy

$$
s_{U}(A \mid B)=\operatorname{Tr} A(\log A-\log B)
$$

as

$$
I(A ; \Phi)=\sup _{E} s_{U}\left(\sum_{n} t_{n} E_{n} \otimes \Phi\left(E_{n}\right) \mid A \otimes \Phi(A)\right)
$$

([16]) which is an extension of (1). Thereby, if a relative matrix entropy corresponding to $s_{U}(A \mid B)$ is realized, then we might extend information entropies to matrix ones. Simply one might think of $A^{\frac{1}{2}}(\log A-\log B) A^{\frac{1}{2}}$ as an observable, but it is probably not suitable since we think a relative entropy should be an initial vector of a geodesic for a certain geometry. In fact, the geodesic of one of the Hiai-Petz geometries [13] is $M_{t}(A, B)=\exp ((1-t) \log A+t \log B)$ and hence its initial tangent vector is expressed by

$$
\begin{aligned}
& \left.\mathfrak{S}_{U}(A \mid B) \equiv \frac{d M_{t}(A, B)}{d t}\right|_{t=0} \\
& =U\left(\left(\frac{1}{\log ^{[1]}\left(d_{i}, d_{j}\right)}\right) \circ U^{*}(\log B-\log A) U\right) U^{*}
\end{aligned}
$$

where $U$ is a unitary with $\operatorname{diag}\left(d_{i}\right)=U^{*} A U$ and the function $f^{[1]}$ is the divided difference $f^{[1]}(x, y)=\frac{f(x)-f(y)}{x-y}$, see $[6,7]$. We think it is a matrix version of the Umegaki entropy. In fact,

$$
\begin{aligned}
& \operatorname{Tr} \mathfrak{S}_{U}(A \mid B) \\
& =\operatorname{Tr} A(\log B-\log A)=-S_{U}(A \mid B) .
\end{aligned}
$$

Also Petz [17] defined a quantum conditional entropy

$$
h\left(\rho_{A B} \mid B\right)=s\left(\rho_{A B}\right)-s(B)
$$

(where a composite matrix $\rho_{A B}=W_{A B}$ will be discussed later) and it is related to the Umegaki entropy:

$$
h\left(\rho_{A B} \mid B\right)=\log \operatorname{dim} H_{A}-s_{U}\left(\rho_{A B} \mid \tau_{A} \otimes \rho_{B}\right)
$$

where $\tau_{A}$ is a tracial state. But $h\left(\rho_{A B} \mid B\right)$ is not always positive unfortunately.

Since the quantum conditional entropy is not positive though it is a numerical quantity and the matrix entropy $\mathfrak{S}_{U}(A \mid B)$ is a somewhat awkward tool, here we do not use $\mathfrak{S}_{U}(A \mid B)$ while we fully use the above idea, in particular, Ohya's construction. In this paper, we use the relative operator entropy

$$
S(A \mid B)=A^{\frac{1}{2}} \log \left(A^{-\frac{1}{2}} B A^{-\frac{1}{2}}\right) A^{\frac{1}{2}}
$$

introduced in [9] and try to extend information entropies to matrix ones.

\section{Relative Operator Entropy}

To begin with, we review the relative operator entropy for positive (bounded linear) operators on a Hilbert space, see $[9,10,11,6]$. Let $A \#_{t} B$ be a weighted geometric operator mean in the sense of Kubo-Ando [15];

$$
A \#_{t} B=A^{\frac{1}{2}}\left(A^{-\frac{1}{2}} B A^{-\frac{1}{2}}\right)^{t} A^{\frac{1}{2}}
$$

for invertible $A$. If $A$ is not invertible, we may define as $A \#_{t} B=\lim _{n \rightarrow \infty}\left(A+\frac{1}{n}\right) \#_{t} B$ in the strong operator topology. In $[10,9]$, we introduced the relative operator entropy $S(A \mid B)$ as a derivative for a differentiable path of geometric operator means $A \#_{t} B$ if the following strong-limit exists as a bounded operator;

$$
\lim _{t \rightarrow 0} \frac{A \#_{t} B-A}{t}
$$

If $B$ is invertible, then

$$
S(A \mid B)=B^{\frac{1}{2}} \eta\left(B^{-\frac{1}{2}} A B^{-\frac{1}{2}}\right) B^{\frac{1}{2}}
$$

where $\eta$ is the entropy function:

$$
\eta(x)=-x \log x \text { if } x>0, \quad \eta(0)=0 .
$$

In addition, if $A$ is invertible, then 


$$
S(A \mid B)=A^{\frac{1}{2}} \log \left(A^{-\frac{1}{2}} B A^{-\frac{1}{2}}\right) A^{\frac{1}{2}}
$$

So the existence condition is;

Lemma 1. If $A$ is majorized by $B$, i.e., $A \leq \alpha B$ for some $\alpha>0$, then $S(A \mid B)$ exists.

In fact, by Douglas' majorization theorem [5], we have $A^{\frac{1}{2}}=D B^{\frac{1}{2}}$ for some operator $D$ and

$$
S(A \mid B)=B^{\frac{1}{2}} \eta\left(D^{*} D\right) B^{\frac{1}{2}} \text {. }
$$

To discuss matrix entropies based on $S(A \mid B)$, we summarize its properties $[8,9,10]$ :

Lemma 2. The relative operator entropy has the following properties if it exists:

(1) If $B \leq B^{\prime}$, then $S(A \mid B) \leq S\left(A \mid B^{\prime}\right)$.

(2) $T^{*} S(A \mid B) T \leq S\left(T^{*} A T \mid T^{*} B T\right)$

(the equality holds for invertible $T$ ).

(2') $S(\alpha A \mid \alpha B)=\alpha S(A \mid B))$ for $\alpha>0$.

(3) $S\left(A_{1} \mid B_{1}\right)+S\left(A_{2} \mid B_{2}\right) \leq S\left(A_{1}+A_{2} \mid B_{1}+B_{2}\right)$.

(3') $(1-t) S\left(A_{1} \mid B_{1}\right)+t S\left(A_{2} \mid B_{2}\right)$

$$
\leq S\left((1-t) A_{1}+t A_{2} \mid(1-t) B_{1}+t B_{2}\right)
$$

for all $t \in[0,1]$.

(4) $S\left(\oplus_{k} A_{k} \mid \oplus_{k} B_{k}\right)=\oplus_{k} S\left(A_{k} \mid B_{k}\right)$.

(4') $S\left(\sum_{k} E_{k} \otimes A_{k} \mid \sum_{k} E_{k} \otimes B_{k}\right)$

$$
=\sum_{k} E_{k} \otimes S\left(A_{k} \mid B_{k}\right) \quad \text { for a } P V M\left\{E_{k}\right\} .
$$

(5) $S(A \mid B) \leq B-A$.

(6) $S(A \mid \alpha B)=(\log \alpha) A+S(A \mid B)$ for $\alpha>0$.

The properties (2), (2'), (3), (3') and (4) are called transformer inequality, homogeneity, subadditivity, joint concavity and orthogonality respectively.

\section{Matrix Probability Space}

From now on, we assume that the space is finite dimensional, that is, operators are matrices. Assume that $A \in \mathcal{M}_{n}^{+}$, the $n \times n$ positive-definite matrices and $B \in \mathcal{M}_{m}^{+}$, the $m \times m$ ones. Let $\left\{E_{k}\right\}$ be the (fixed) decomposition of the identity, that is, each $E_{k}$ be a projection and $\sum_{k} E_{k}=I_{n}$. A set $\left\{E_{k}\right\}$ is considered as elementary probability events. Let $A=\sum_{k} t_{k} E_{k}$ be a spectral decomposition of of an invertible density matrix, that is, is positive-definite and $\operatorname{Tr} A=1$. Then, we can observe that the probability $p\left(E_{k}\right)$ is given by $\operatorname{Tr}\left(t_{k} E_{k}\right)=t_{k} \operatorname{Tr}\left(E_{k}\right)$.

Let $\Phi$ be a quantum channel from $\mathcal{M}_{n}$ to $\mathcal{M}_{m}$. Then each $F_{k}=\Phi\left(E_{k}\right)$ is considered as an elementary event, but it is no longer a projection. So we take a fixed POVM $\left\{F_{\ell}\right\}$, and consider a density matrix $B=\sum_{\ell} s_{\ell} F_{\ell}$. Assume $s_{\ell}>0$. Then note that $B$ is invertible since

$B \geq \sum_{\ell} \min _{j}\left\{s_{j}\right\} F_{\ell}=\min _{j}\left\{s_{j}\right\} I_{m}$.

In this situation, we define a composite matrix $W_{A B}$ for $A$ and $B$ by

$$
\sum_{k, \ell} w_{k \ell} E_{k} \otimes F_{\ell}
$$

where

$w_{k \ell} \geq 0, \sum_{k} w_{k \ell} \operatorname{Tr} E_{k}=s_{\ell}, \sum_{\ell} w_{k \ell} \operatorname{Tr} F_{\ell}=t_{k} . \quad$ In fact, for the partial traces $\operatorname{Tr}_{1}$ and $\operatorname{Tr}_{2}$ for each term, we have

$$
\operatorname{Tr}_{2}\left(W_{A B}\right)=\sum_{k} \operatorname{Tr} \sum_{\ell} w_{k \ell} F_{\ell} E_{k}=\sum_{k} t_{k} E_{k}=A
$$

and similarly $\operatorname{Tr}_{1}\left(W_{A B}\right)=B$, so that $A$ and $B$ are the 'marginal distributions'. The composite probability is $p\left(E_{k} \otimes F_{\ell}\right)=w_{k \ell} \operatorname{Tr} E_{k} \operatorname{Tr} F_{\ell}$, and hence the conditional ones are as follows:

$$
\begin{aligned}
& p\left(E_{k} \otimes F_{\ell} \mid F_{\ell}\right)=\frac{w_{k \ell} \operatorname{Tr} E_{k}}{s_{\ell}}, \\
& p\left(E_{k} \otimes F_{\ell} \mid E_{k}\right)=\frac{w_{k \ell} \operatorname{Tr} F_{\ell}}{t_{k}} .
\end{aligned}
$$

A typical example for $W_{A B}$ is $\sum_{k, \ell} t_{k} s_{\ell} E_{k} \otimes F_{\ell}$. In this case, $A$ and $B$ are considered independent. In fact, in this case, $p\left(E_{k} \otimes F_{\ell}\right)=p\left(E_{k}\right) p\left(F_{\ell}\right)$ holds for each $k, \ell$. The following example is not independent:

Example 1. Let $\left(w_{i j}\right)$ be given by a nonnegative 
matrix

$$
\left(w_{k \ell}\right)=\frac{1}{12}\left(\begin{array}{ll}
6 & 1 \\
2 & 3
\end{array}\right)
$$

and all $E_{k}$ and $F_{k}$ projections of rank one. Then we have the marginal probabilities

$$
t_{1}=\frac{7}{12}, t_{2}=\frac{5}{12}, s_{1}=\frac{2}{3}, s_{2}=\frac{1}{3} .
$$

The are not independent since, for instance, $w_{11}=\frac{1}{2} \neq \frac{14}{36}=t_{1} s_{1}$. For positive semidefinite matrices of rank one

$$
\begin{gathered}
E_{1}=\left(\begin{array}{ll}
1 & 0 \\
0 & 0
\end{array}\right), \quad E_{2}=\left(\begin{array}{ll}
0 & 0 \\
0 & 1
\end{array}\right), \\
F_{1}=\frac{1}{5}\left(\begin{array}{ll}
4 & 2 \\
2 & 1
\end{array}\right), \quad F_{2}=\frac{1}{5}\left(\begin{array}{cc}
1 & -2 \\
-2 & 4
\end{array}\right),
\end{gathered}
$$

we have the marginal density matrices

$$
\begin{gathered}
A=\frac{7}{12} E_{1}+\frac{5}{12} E_{2}=\frac{1}{12}\left(\begin{array}{ll}
7 & 0 \\
0 & 5
\end{array}\right), \\
B=\frac{2}{3} F_{1}+\frac{1}{3} F_{2}=\frac{1}{15}\left(\begin{array}{ll}
9 & 2 \\
2 & 6
\end{array}\right),
\end{gathered}
$$

and the composite matrix

$$
\begin{aligned}
W_{A B} & =\left(\begin{array}{ccc}
\frac{6}{12} F_{1}+\frac{1}{12} F_{2} & 0 \\
0 & \frac{2}{12} F_{1}+\frac{3}{12} F_{2}
\end{array}\right) \\
& =\frac{1}{60}\left(\begin{array}{cccc}
25 & 10 & 0 & 0 \\
10 & 10 & 0 & 0 \\
0 & 0 & 11 & -2 \\
0 & 0 & -2 & 14
\end{array}\right) .
\end{aligned}
$$

\section{Matrix Entropies}

The composite matrix entropy $H\left(W_{A B}\right)$ is defined by $H\left(W_{A B}\right)=\eta\left(W_{A B}\right)$. Also the mutual matrix entropy by $I(A ; B)$ and the conditional entropies $H\left(W_{A B} \mid A\right)$ and $H\left(W_{A B} \mid B\right)$ defined by

$$
\begin{aligned}
I(A ; B) & =-S\left(W_{A B} \mid A \otimes B\right), \\
H\left(W_{A B} \mid A\right) & =S\left(W_{A B} \mid A \otimes I\right), \\
H\left(W_{A B} \mid B\right) & =S\left(W_{A B} \mid I \otimes B\right) .
\end{aligned}
$$

Note that these matrices can be defined since $A$ and $B$ are invertible (see Lemma exist). Immediately we have $H\left(W_{A B}\right) \geq 0$ and $H\left(W_{A B} \mid B\right) \geq 0$, while $I(A ; B)$ is not always positive-semidefinite (see Example ex2 in the below) since the individual mutual information may be negative. But its trace is positive:

Lemma 3. $\operatorname{Tr} I(A ; B) \geq 0$.

By Lemma 2(5), we have

$$
\begin{aligned}
& \operatorname{Tr} I(A ; B) \geq \operatorname{Tr}\left(\operatorname{Tr}_{2} W_{A B}\right)-\operatorname{Tr} A \otimes B \\
& =\operatorname{Tr} A-(\operatorname{Tr} A)(\operatorname{Tr} B)=1-1=0 .
\end{aligned}
$$

Remark 1. Contrary to the classical case, another mutual matrix entropy $I(B ; A)$, even if it is defined well, is not equal to $I(A ; B)$ since

$A \otimes B \neq B \otimes A$ and the usual product also is not commuting.

Then, by Lemma 2(4'), we express these entropies as follows:

Lemma 4. Matrix entropies have the following decompositions:

$$
\begin{gathered}
H\left(W_{A B}\right)=\sum_{k} E_{k} \otimes \eta\left(\sum_{l} w_{k l} F_{l}\right) . \\
I(A ; B)=-\sum_{k} E_{k} \otimes S\left(\sum_{l} w_{k l} F_{l} \mid t_{k} B\right) \\
=-\sum_{k, l} \log t_{k} E_{k} \otimes w_{k l} F-\sum_{k} E_{k} \otimes S\left(\sum_{l} w_{k l} F_{l} \mid t_{k} B\right) \\
H\left(W_{A B} \mid A\right)=\sum_{k} E_{k} \otimes S\left(\sum_{l} w_{k l} F_{l} \mid t_{k} I\right) \\
=\sum_{k} t_{k} E_{k} \otimes \eta\left(\sum_{l} \frac{w_{k l}}{t_{k}} F_{l}\right) . \\
H\left(W_{A B} \mid A\right)=\sum_{k} E_{k} \otimes S\left(\sum_{l} w_{k l} F_{l} \mid B\right) .
\end{gathered}
$$

In addition, all $F_{\ell}$ are (mutually orthogonal) projections, then

$$
\begin{gathered}
H\left(W_{A B}\right)=\sum_{k, \ell} \eta\left(w_{k \ell}\right) E_{k} \otimes F_{\ell} . \\
I(A ; B)=-\sum_{k, \ell} w_{k \ell} \log \frac{t_{k} s_{\ell}}{w_{k \ell}} E_{k} \otimes F_{\ell} . \\
H\left(W_{A B} \mid A\right)=\sum_{k, \ell} w_{k \ell} \log \frac{t_{k}}{w_{k \ell}} E_{k} \otimes F_{\ell} . \\
H\left(W_{A B} \mid B\right)=\sum_{k, \ell} w_{k \ell} \log \frac{s_{\ell}}{w_{k \ell}} E_{k} \otimes F_{\ell} .
\end{gathered}
$$

Thus, the latter case, where $\left(\left\{F_{\ell}\right\}\right.$ is a PVM, 
shows the entropy values in the classical (commutative) case. So we confirm easily that $I(A ; B)$ is not always positive even for diagonal matrices corresponding to the classical ones:

Example 2. We simplify Example 1; $F_{1}=E_{1}$, $F_{2}=E_{2}$ and $B=\frac{1}{3}\left(\begin{array}{ll}2 & 0 \\ 0 & 1\end{array}\right)$. By the above lemma (2), we have only to show $S\left(\sum_{\ell} w_{1 \ell} F_{\ell} \mid t_{1} B\right)$ is neither positive nor negative:

$$
\begin{aligned}
& S\left(\frac{1}{12}\left(\begin{array}{ll}
6 & 0 \\
0 & 1
\end{array}\right) \mid \frac{7}{36}\left(\begin{array}{ll}
2 & 0 \\
0 & 1
\end{array}\right)\right) \\
= & \frac{1}{36} S\left(\left(\begin{array}{cc}
18 & 0 \\
0 & 3
\end{array}\right) \mid\left(\begin{array}{cc}
14 & 0 \\
0 & 7
\end{array}\right)\right) \\
= & \frac{1}{36}\left(\begin{array}{cc}
18 \log _{\frac{14}{18}} & 0 \\
0 & 3 \log \frac{7}{3}
\end{array}\right) .
\end{aligned}
$$

Since $\log \frac{14}{18}<0$ and $\log \frac{7}{3}>0$, we have the required result. Also (2') shows this;

$$
\log \frac{w_{11}}{t_{1} s_{1}}=\log \frac{9}{7}>0 \text { and } \log \frac{w_{12}}{t_{1} s_{2}}=\log \frac{3}{7}<0 .
$$

In the context for the composite elementary events $\left\{E_{k} \otimes F_{\ell}\right\}$, the entropy $\eta(A), \eta(B)$ should be extended to

$$
\begin{aligned}
& H_{F}(A)=-\sum_{k, \ell} \log \left(t_{k}\right) w_{k \ell} E_{k} \otimes F_{\ell}, \text { and } \\
& H_{E}(B)=-\sum_{k, \ell} \log \left(s_{\ell}\right) w_{k \ell} E_{k} \otimes F_{\ell} .
\end{aligned}
$$

In fact, we obtain

$$
\begin{aligned}
\operatorname{Tr}_{2}\left(H_{F}(A)\right) & =-\sum_{k} \operatorname{Tr}\left(w_{k \ell} F_{\ell}\right) \log \left(t_{k}\right) E_{k} \\
= & -\sum_{k} t_{k} \log \left(t_{k}\right) E_{k} \\
& =\sum_{k} \eta\left(t_{k}\right) E_{k}=\eta(A)
\end{aligned}
$$

and similarly $\operatorname{Tr}_{1}\left(H_{E}(B)\right)=\eta(B)$. Then we have

$$
\begin{aligned}
& H\left(W_{A B}\right) \\
& =\frac{1}{12}\left(\begin{array}{c}
(6 \log 2) F_{1}+(\log 12) F_{2} \\
0
\end{array}\right.
\end{aligned}
$$

the following relations similar to the classical cases:

Theorem 5. The following equalities hold:

(1) $H\left(W_{A B} \mid B\right)+I(A ; B)=H_{F}(A)$

(2) $H_{F}(A)+H\left(W_{A B} \mid A\right)=H\left(W_{A B}\right)$.

Proof. By Lemma 4 and Lemma 2 (6), we have (1) as follows:

$$
\begin{aligned}
& H\left(W_{A B} \mid B\right)+I(A ; B) \\
& =\sum_{k} E_{k} \otimes S\left(\sum_{l} w_{k \ell} F_{\ell} \mid B\right) \\
& -\sum_{k} E_{k} \otimes S\left(\sum_{l} w_{k \ell} F_{\ell} \mid t_{k} B\right) \\
& =\sum_{k} E_{k} \otimes\left[S\left(\sum_{l} w_{k \ell} F_{\ell} \mid B\right)\right. \\
& =-\left(\log t_{k}\right)\left(\sum_{l} w_{k \ell} F_{\ell}-S\left(\sum_{l} w_{k \ell} F_{\ell} \mid t_{k} B\right)\right] \\
& \sum_{k, l} \log \left(t_{k}\right) w_{k \ell} E_{k} \otimes F_{\ell}=H_{F}(A) .
\end{aligned}
$$

Similarly (2) follows from

$H_{F}(A)+H\left(W_{A B} \mid B\right)=$

$-\sum_{k, l} \log \left(t_{k}\right) w_{k \ell} E_{k} \otimes F+\sum_{k} E_{k} \otimes S\left(\sum_{l} w_{k \ell} F_{\ell} \mid t_{k} I\right)$

$=\sum_{k} E_{k} \otimes \eta\left(\sum_{l} w_{k \ell} F_{\ell}\right)$

$=\eta\left(\sum_{k} E_{k} \otimes \sum_{l} w_{k \ell} F_{l}\right)$

$=\eta\left(\sum_{k, l} w_{k \ell} E_{k} \otimes F_{l}\right)=\eta\left(W_{A B}\right)=H\left(W_{A B}\right)$.

Remark 2. As in the above, $H\left(W_{A B} \mid A\right)+I(A ; B)$ is not equal to $H_{E}(B)$ in general.

Example 3. In Example 1, we exchange $\left\{f_{\ell}\right\}$ to some POVM. Then the following matrix entropies similar to the classical ones as in Example 2:
$\left.\begin{array}{c}0 \\ (2 \log 6) F_{1}+(3 \log 4) F_{2}\end{array}\right)$ 


$$
\begin{aligned}
& I(A ; B) \\
& =\frac{1}{12}\left(\begin{array}{cc}
\left(6 \log _{\frac{9}{7}}\right) F_{1}+\left(\log _{\frac{3}{7}}\right) F_{2} & 0 \\
0 & \left(2 \log _{\frac{3}{5}}\right) F_{1}+\left(3 \log \frac{9}{5}\right) F_{2}
\end{array}\right), \\
& H\left(W_{A B} \mid A\right) \\
& =\frac{1}{12}\left(\begin{array}{cc}
\left(6 \log _{\frac{7}{6}}\right) F_{1}+(\log 7) F_{2} & 0 \\
0 & \left(2 \log _{\frac{5}{2}}\right) F_{1}+\left(3 \log _{\frac{5}{3}}\right) F_{2}
\end{array}\right), \\
& H\left(W_{A B} \mid B\right) \\
& =\frac{1}{12}\left(\begin{array}{cc}
\left(6 \log \frac{4}{3}\right) F_{1}+(\log 4) F_{2} & 0 \\
0 & (2 \log 4) F_{1}+(3 \log 2) F_{2}
\end{array}\right), \\
& H_{F}(A) \\
& =\frac{1}{12}\left(\begin{array}{cc}
\left(6 \log \frac{12}{7}\right) F_{1}+\left(\log \frac{12}{7}\right) F_{2} & 0 \\
0 & \left(2 \log \frac{12}{5}\right) F_{1}+\left(3 \log \frac{12}{5}\right) F_{2}
\end{array}\right) .
\end{aligned}
$$

Similar identities hold like classical cases for a $\operatorname{PVM}\left\{F_{\ell}\right\}$ :

$$
\begin{aligned}
& \sum_{\ell} w_{1 \ell} F_{\ell}=\frac{6 F_{1}}{12}+\frac{F_{2}}{12}=\frac{9}{48} P_{1}+\frac{19}{48} P_{2}, \\
& \sum_{\ell} w_{2 \ell} F_{\ell}=\frac{2 F_{1}}{12}+\frac{3 F_{2}}{12}=\frac{11}{48} P_{1}+\frac{9}{48} P_{2},
\end{aligned}
$$

Corollary 6. If $\left\{F_{\ell}\right\}$ is a PVM, then

(1) $H\left(W_{A B} \mid A\right)+I(A ; B)=H_{E}(B)$, and

(2) $H_{E}(B)+H\left(W_{A B} \mid B\right)=H\left(W_{A B}\right)$.

In the above corollary, the assumption are necessary as in the following example:

Example 4. In the situation of Example 3, consider the case that each $F_{k}$ is not a projection: Let

$$
\begin{gathered}
P_{1}=\frac{1}{2}\left(\begin{array}{cc}
1 & -1 \\
-1 & 1
\end{array}\right), P_{2}=\frac{1}{2}\left(\begin{array}{ll}
1 & 1 \\
1 & 1
\end{array}\right), \\
F_{1}=\frac{1}{4} P_{1}+\frac{3}{4} P_{2}=\frac{1}{4}\left(\begin{array}{ll}
2 & 1 \\
1 & 2
\end{array}\right) \text { and } \\
F_{2}=\frac{3}{4} P_{1}+\frac{1}{4} P_{2}=\frac{1}{4}\left(\begin{array}{cc}
2 & -1 \\
-1 & 2
\end{array}\right) .
\end{gathered}
$$

Then $B=\frac{2}{3} F_{1}+\frac{1}{3} F_{2}=\frac{1}{12}\left(\begin{array}{ll}6 & 1 \\ 1 & 6\end{array}\right)=\frac{5 P_{1}}{12}+\frac{7 P_{2}}{12}$

we have

$$
\begin{aligned}
& S\left(\sum_{l} w_{1 l} F_{\ell} \mid t_{1} I\right)=\frac{9}{48} \log \frac{28}{9} P_{1}+\frac{19}{48} \log \frac{28}{19} P_{2}, \\
& S\left(\sum_{l} w_{2 l} F_{\ell} \mid t_{2} I\right)=\frac{11}{48} \log \frac{20}{11} P_{1}+\frac{9}{48} \log \frac{20}{9} P_{2}
\end{aligned}
$$

and hence $H\left(W_{A B} \mid A\right)$

$$
\begin{aligned}
&=\left.\begin{array}{rr}
\frac{9}{48} \log \frac{28}{9} P_{1}+\frac{19}{48} \log \frac{28}{19} P_{2} & \\
& \frac{11}{48} \log \frac{20}{11} P_{1}+\frac{9}{48} \log \frac{20}{9} P_{2}
\end{array}\right) . \\
& \text { Since } t_{1} B=\frac{35 P_{1}+49 P_{2}}{144} \text { and } t_{2} B=\frac{25 P_{1}+35 P_{2}}{144}, \text { we }
\end{aligned}
$$
have $I(A ; B)$

and

$$
\begin{aligned}
& H_{E}(B)=\frac{1}{12}\left(\begin{array}{ll}
6 \log \frac{3}{2} F_{1}+\log 3 F_{2} & \\
& 2 \log \frac{3}{2} F_{1}+3 \log 3 F_{2}
\end{array}\right) \\
& =\left(\begin{array}{ll}
\frac{1}{16} \log \frac{3^{3}}{2^{2}} P_{1}+\frac{1}{48} \log \frac{3^{19}}{2^{18}} P_{2} & \\
& \frac{1}{48} \log \frac{3^{11}}{2^{2}} P_{1}+\frac{1}{16} \log \frac{3^{3}}{2^{2}} P_{2}
\end{array}\right) \text {. }
\end{aligned}
$$

By

$$
=-\left(\begin{array}{ll}
\frac{9}{48} \log \frac{35}{27} P_{1}+\frac{19}{48} \log \frac{49}{57} P_{2} & \\
& \frac{11}{48} \log \frac{25}{33} P_{1}+\frac{9}{48} \log \frac{35}{27} P_{2}
\end{array}\right),
$$

we have $H\left(W_{A B} \mid A\right)+I(A ; B)$

$=\left(\begin{array}{ll}\frac{9}{48} \log \frac{12}{5} P_{1}+\frac{19}{48} \log \frac{12}{7} P_{2} & \\ & \frac{11}{48} \log \frac{12}{5} P_{1}+\frac{9}{48} \log \frac{12}{7} P_{2}\end{array}\right)$, 
which does not coincides with $H_{E}(B)$.

\section{References}

[1] E. Andruchow, G. Corach and D. Stojanoff: Geometrical significance of Lowner-Heinz inequality, Proc. Amer. Math. Soc., 128 (2000), 1031-1037.

[2] V.P. Belavkin, P. Staszewski: $C^{*}$-algebraic generalization of relative entropy and entropy, Ann. Inst. H. Poincare Sect. A.37(1982), 51-58.

[3] G. Corach, H. Porta and L. Recht: Geodesics and operator means in the space of positive operators. Internat. J. Math. 4 (1993), 193-202.

[4] G. Corach and A.L.Maestripieri: Differential and metrical structure of positive operators, Positivity 3 (1999), 297-315.

[5] R.G. Douglas: On majorization, factorization and range inclusion of operators in Hilbert space, Proc. Amer. Math. Soc., 17 (1966), 413-416.

[6] J. I. Fujii: The Hiai-Petz geodesic for strongly convex norm is the unique shortest path, Sci. Math. Japon. Online, e-2009 (2009), 579-586.

[7] J. I. Fujii: Structure of Hiai-Petz parametrized geometry for positive definite matrices, Linear Algebra. Appl. 432 (2010), 318-326.

[8] J. I. Fujii, M. Fujii and Y. Seo: An extension of the Kubo -Ando theory : Solidarities, Math. Japon., 35 (1990), 509-512.
[9] J. I. Fujii and E. Kamei: Relative operator entropy in noncommutative information theory, Math. Japon. 34 (1989), 341-348.

[10] J.I.Fujii and E.Kamei: Uhlmann's interpolational method for operator means, Math. Japon. 34 (1989), 541--547.

[11] J. I. Fujii and E. Kamei: Interpolational paths and their derivatives, Math. Japon. 39 (1993), 557-560.

[12] F. Hiai and H. Kosaki: Means of Hilbert space operators, Lecture Notes in Math., vol. 1820 (2003), Springer-Verlag.

[13] F. Hiai and D. Petz: Riemannian metrics on positive definite matrices related to means, Linear Alg. Appl. 430 (2009), 3105-3130.

[14] R.A. Horn and C. R.Johnson: Topics in Matrix Analysis, Cambridge Univ. Press, 1991.

[15] F. Kubo and T. Ando: Means of positive linear operators, Math. Ann. 246 (1980), 205-224.

[16] M. Ohya, On compound state and mutual information in quantum information theory, IEEE trans. IT, 29(1983), 770-774.

[17] D. Petz, Quantum Information Theory and Quantum Statistics', Springer, 2008.

[18] Uhlmann: Relative entropy and the Wigner-YamaseDyson-Lieb concavity in an interpolation theory, Commun. Math. Phys. 54 (1977), 22-32.

[19] H. Umegaki: Conditional expectation in an operator algebra IV, Kodai Math. Sem. Rep. 14 (1962), 59-85. 